\title{
Dedicated immunosensing of the mouse intestinal epithelium facilitated by a pair of genetically coupled lectin-like receptors
}

\author{
S Leibelt ${ }^{1}$, ME Friede $^{1}$, C Rohe ${ }^{1}$, D Gütle ${ }^{2}$, E Rutkowski $^{1}$, A Weigert $^{3}$, L Kveberg $^{4}$, JT Vaage $^{4}$, MW Hornef $^{2}$ \\ and A Steinle ${ }^{1}$
}

The integrity of the intestinal epithelium is constantly surveyed by a peculiar subset of innate-like T lymphocytes embedded in the epithelial cell layer, hence called intestinal intraepithelial lymphocytes (IELs). IELs are thought to act as "first-line" sentinels sensing the state of adjacent epithelial cells via both T-cell receptors and auxiliary receptors. Auxiliary receptors modulating IEL activity include C-type lectin-like receptors encoded in the natural killer gene complex such as NKG2D. Here, we report that the CTLR Nkrp1g is expressed by a subpopulation of mouse CD103 ${ }^{+}$IELs allowing immunosensing of the intestinal epithelium through ligation of the genetically coupled CTLR Clr-f that is almost exclusively expressed on differentiated intestinal epithelial cells (IECs). Most of these Nkrp1g-expressing IELs exhibit a $\gamma \delta \mathrm{TCR}^{\text {bright }} \mathrm{Nkg} 2 \mathrm{a}^{-}$phenotype and are intimately associated with the intestinal epithelium. As Clr-f expression strongly inhibits effector functions of Nkrp1g-expressing cells and is upregulated upon poly(l:C) challenge, Clr-f molecules may quench reactivity of these IELs towards the epithelial barrier that is constantly provoked by microbial and antigenic stimuli. Altogether, we here newly characterize a genetically linked C-type lectin-like receptor/ligand pair with a highly restricted tissue expression that apparently evolved to allow for a dedicated immunosurveillance of the mouse intestinal epithelium.

\section{INTRODUCTION}

Mammalian epithelia cover body surfaces directly exposed to the environment and are populated by a complex community of commensal microorganisms. In addition, these epithelia are constantly challenged by potential pathogens. Hence, epithelial cells not only constitute a physical barrier of skin, intestine, and other exposed organs, but also are actively involved in pathogen recognition and immune defense. ${ }^{1-3}$ In the intestine, a single thin layer of intestinal epithelial cells (IECs) protects a huge area $\left(\sim 200 \mathrm{~m}^{2} \text { in humans) }\right)^{4}$ against pathogenic microorganisms also comprising specialized types of cells that produce protective mucus or secrete anti-microbial peptides. ${ }^{2,5,6}$ Although most host-microbiota interactions are of symbiotic nature, the immense number and density of bacteria in the gut constitutes a particular challenge for the immune system. Therefore, pathogen recognition and subsequent initiation and modulation of immune responses are major tasks of IECs administered in a complex network together with innate and adaptive immune cells. A peculiar subset of the latter resides in tight contact with IECs within the epithelial barrier and in close proximity to the intestinal lumen. These so-called intestinal intraepithelial lymphocytes (IELs) act as a first line of defense, mostly comprising antigen-experienced $\alpha \beta \mathrm{T}$ cells or $\gamma \delta \mathrm{T}$ cells. They commonly express activation markers, such as CD69, and the integrin CD103 that mediates gut homing. ${ }^{7}$ Apart from T-cell receptor (TCR) engagement, activity of these IELs is regulated by auxiliary receptors such as killer cell lectin-like receptors (KLRs) encoded in the natural killer gene complex (NKC). ${ }^{8}$ Prominent NKC-encoded KLRs are NK1.1, NKG2D, CD94/NKG2A, and various Ly49 receptors. ${ }^{7,9,10}$ Although NK1.1 (also known as Nkrp1c) is a member of the NKRP1 family with a yet unknown ligand, ${ }^{11}$ other members of the NKRP1 subfamily have been shown to ligate likewise C-type lectin-like receptors (CTLRs) of the CLEC2 family, with genes

${ }^{1}$ Institute for Molecular Medicine, Goethe-University Frankfurt am Main, Frankfurt am Main, Germany. ${ }^{2}$ Institute for Medical Microbiology and Hospital Epidemiology, Hannover Medical School, Hannover, Germany. ${ }^{3}$ Institute for Biochemistry I, Goethe-University Frankfurt am Main, Frankfurt am Main, Germany and ${ }^{4}$ Department of Immunology, Oslo University Hospital, Rikshospitalet and University of Oslo, Oslo, Norway. Correspondence: A Steinle (alexander.steinle@kgu.de) 
of both NKRP1 receptors and CLEC2 ligands intermingled in the NKC. ${ }^{12-15}$ Members of the CLEC2 family are CD69, the human CTLRs LLT1, AICL, KACL, as well as the mouse C-type lectin-related (Clr) molecules. ${ }^{8,11}$ Certain mouse Clr molecules have been shown to act as ligands of NKRP1 receptors expressed by NK cells and T cells, whereas expression and function of most of these Clr molecules are poorly defined. ${ }^{11,12,14-17}$ Also, function and immunological relevance of these genetically linked receptor/ligand pairs in vivo remain unknown.

Hence, we set out to characterize the expression and function of mouse Clr molecules. In our initial studies, we unexpectedly observed a rather tissue-restricted RNA expression pattern of some, but not all, Clr family members, well in line with a more recent study by Makrigiannis and co-workers. ${ }^{18}$ In particular, Clr-f transcripts were almost exclusively found associated with the mouse intestine prompting further in-depth studies of cellular expression of Clr-f and its receptor Nkrp1g. Using novel antibodies, our subsequent studies revealed colocalized expression of Clr-f glycoproteins and inhibitory Nkrplg receptors within the intestinal epithelium by IECs and adjacent IELs, respectively, suggesting that this receptor/ligand pair contributes to the immunosurveillance of mouse intestinal epithelium in a tissue-specific manner.

\section{RESULTS}

\section{Predominant expression of the mouse CLEC2 family member Clr-f in gut epithelium}

The mouse Clr multigene family clustered in the mouse NKC comprises at least seven members that are predicted to encode for functional proteins. ${ }^{11,13,15}$ As we had recently observed a highly tissue-specific expression of the human CLEC2 family member CLEC2A encoding for the keratinocyte-associated CTLR KACL, ${ }^{19,20}$ we hypothesized that also certain mouse CLEC2 family proteins may be distinguished by a tissuespecific expression pattern. Hence, we set out to determine expression of mouse Clr family members in various tissues by quantitative PCR (qPCR). In line with previous reports, ${ }^{12,16,21,22}$ we found a rather broad and uniform expression of the gene Clec2d (encoding Clr-b) in most tissues except the brain (Figure 1a and Supplementary Figure S1 online). In contrast, expression of Clec $2 i$ (Clr-g) apparently is biased towards hematopoietic organs. Strikingly, the expression pattern of $\mathrm{Clec} 2 \mathrm{~h}$ (Clr-f) turned out to be completely different from that of both $\mathrm{Clr}-\mathrm{b}$ and $\mathrm{Clr}-\mathrm{g}$ with a predominant expression in the gut, including both small and large intestine (Figure 1a). Significant Clr-f expression was also noted for kidneys, whereas in all the other organs Clr-f transcripts were sparse or not even detectable. This peculiar gut-restricted expression pattern of $\mathrm{Cl}$ - $\mathrm{f}$ was consistently observed for both male and female mice, as well as for $\mathrm{C} 57 \mathrm{BL} / 6$ and $\mathrm{BALB} / \mathrm{c}$ mice (Figure 1a and b). Sectional analyses of the gastrointestinal tract revealed a rather uniform $\mathrm{Clr}-\mathrm{f}$ expression throughout all parts of the small intestine and colon, whereas no expression was found in the esophagus or stomach (Figure 1c). To define the type of intestinal cells specifically expressing Clr-f, small intestines of C57BL/6 mice were dissociated and populations of IECs and IELs purified by FACSorting, respectively (Supplementary Figure S2). Subsequent qPCR analyses revealed a virtually exclusive expression of Clr-f by IECs (Figure 1d), which was unexpected given the common expression of human and mouse CLEC2 family members by hematopoetic cells.
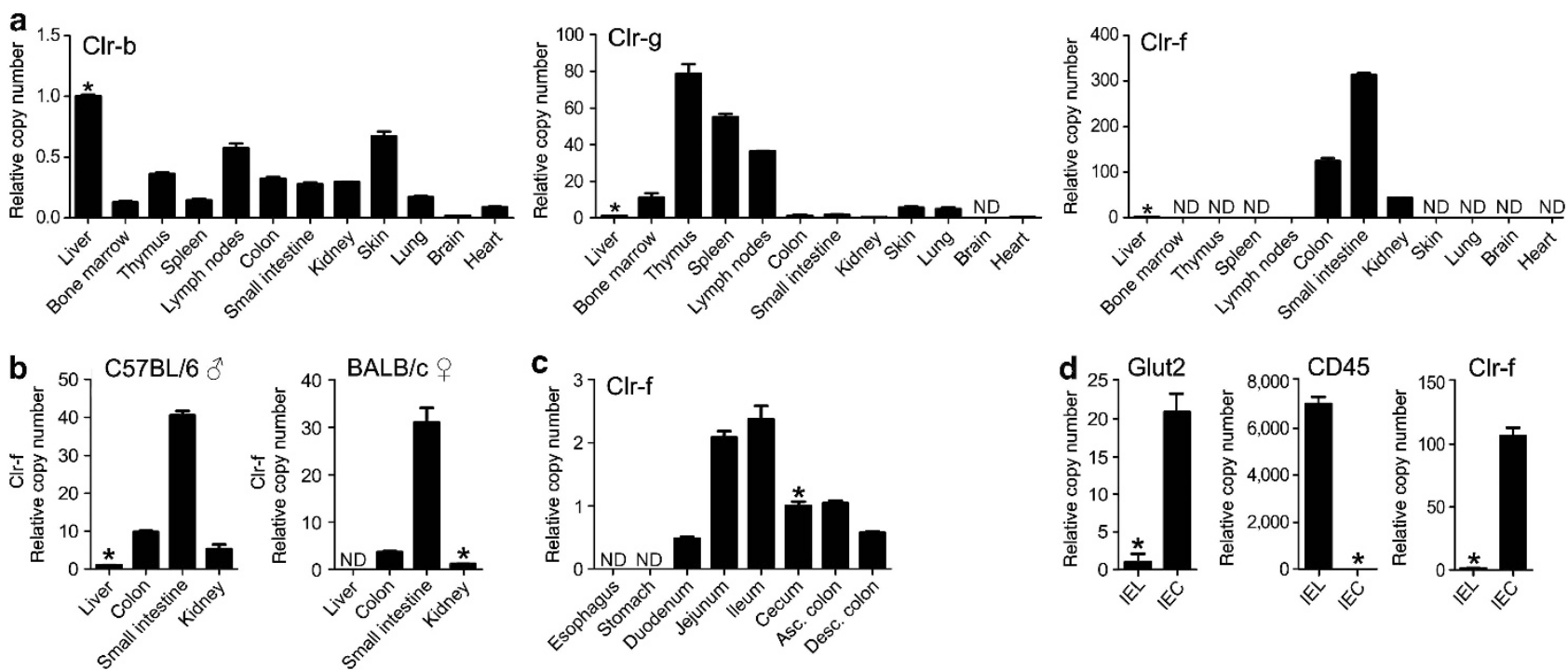

Figure 1 Tissue-specific expression of Clr-f. (a) Relative abundance of Clr-b (left), Clr-g (middle), and Clr-f (right) transcripts, respectively, in various tissues of female C57BL/6 mice. (b and c) Relative abundance of Clr-f transcripts (b) in selected tissues of male C57BL/6 mice (left), female BALB/c mice (right), or (c) in various sections of the gastrointestinal tract of female C57BL/6 mice. (d) Levels of Clr-f transcripts in FACSorted IEL

$\left(\mathrm{CD} 45.2^{+} \mathrm{CD}_{103^{+}} \mathrm{EpCAM}^{-}\right)$and IEC (CD45.2 $\left.{ }^{-} \mathrm{EpCAM}^{+}\right)$, respectively. Purity of IEL and IEC sorted from the crude preparations of intestinal cells was verified by analysis of transcripts of CD45 and Glut2, respectively. (a-d) Levels of transcripts were determined by qPCR, normalized with 18S rRNA, and arbitrarily set relative to transcript levels of respective tissues marked with an asterisk (ND, not detectable). Asc. colon, ascending colon; Clr, C-type lectinrelated; desc. colon, descending colon; IEC, intestinal epithelial cell; IEL, intraepithelial lymphocyte; qPCR, quantitative polymerase chain reaction. 

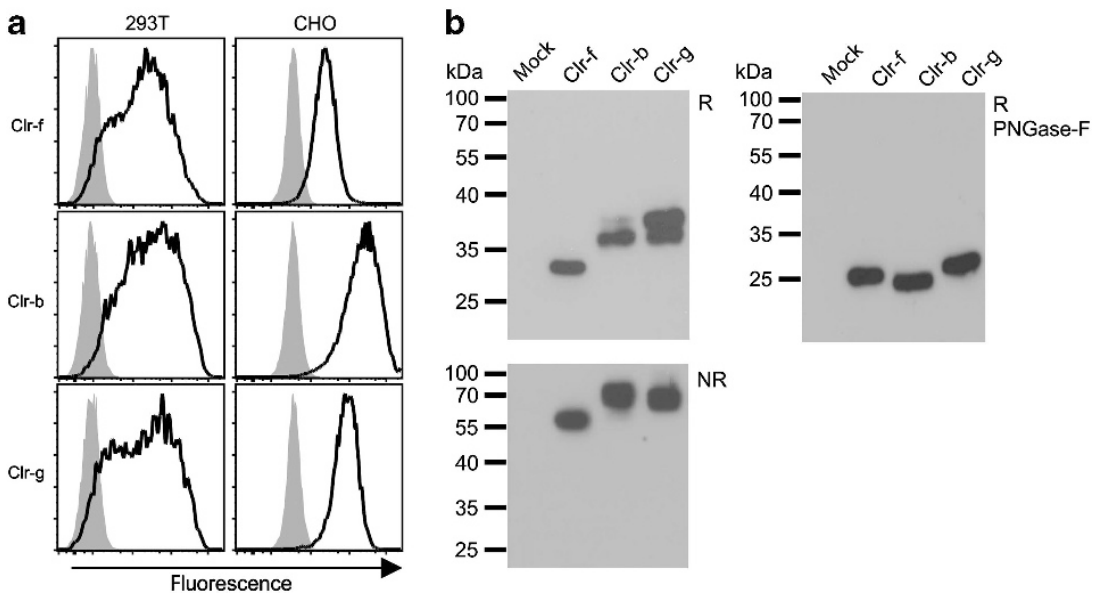

Figure 2 Clr-f is a disulfide-linked homodimeric CTLR. (a) Surface expression of Clr molecules on 293T (left) and CHO cells (right) transfected with FLAG-tagged Clr-f, Clr-b, or Clr-g cDNA detected by FLAG-tag mAb M2 (solid line; shaded: isotype staining). (b) Immunoblotting of lysates of 293T transfected with FLAG-tagged Clr-f, Clr-b, or Clr-g cDNA. Clr molecules were detected by FLAG-tag mAb M2 after reducing SDS-PAGE (SDS-polyacrylamide gel electrophoresis upper left), after reducing SDS-PAGE of PNGase-F-treated lysates (upper right) or after nonreducing SDS-PAGE (lower). Clr, C-type lectin-related; CTLR, C-type lectin-like receptor.

\section{Clr-f is a bona fide homodimeric cell surface CTLR}

To explore whether Clr-f represents a typical cell surface CTLR, the putative open reading frame cloned from cDNA of small intestines was ectopically expressed in $293 \mathrm{~T}$ and $\mathrm{CHO}$ cells. Flow cytometric analyses of the resulting transfectants showed that $\mathrm{Clr}-\mathrm{f}$ is readily expressed at the cell surface comparable to $\mathrm{Clr}-\mathrm{b}$ and Clr-g (Figure 2a). Immunoblots of corresponding 293T lysates revealed glycoproteins with molecular masses of $\sim 30 \mathrm{kDa}(\mathrm{Clr}-\mathrm{f})$ and $\sim 35 \mathrm{kDa}(\mathrm{Clr}-\mathrm{b}$; Clr-g), respectively (Figure 2b). Deglycosylation of all proteins reduced the apparent molecular masses to $\sim 25 \mathrm{kDa}$ (Figure $2 \mathbf{b}$ ) matching the predicted molecular masses and indicating the usage of one (Clr-f), three (Clr-b) or three to four (Clr-g) N-linked glycosylation sites, respectively. Similar results were also obtained when Clr-f was expressed in the human intestinal epithelial cell line HCT116 (Supplementary Figure S3). Under nonreducing conditions, these glycosylated CTLRs appeared with molecular masses of $\sim 60 \mathrm{kDa}(\mathrm{Clr}-\mathrm{f})$ and $\sim 70 \mathrm{kDa}$ (Clr-b, Clr-g), respectively (Figure 2b), demonstrating occurrence as disulfide-linked homodimers.

\section{Selective expression of Clr-f on differentiated intestinal epithelial cells}

Detection of endogenous Clr-f expression was hampered by the lack of Clr-f-specific antibodies. Hence, we expressed the soluble C-type lectin-like domain (CTLD) of Clr-f (sClr-f) in 293 cells and immunized rats with purified sClr-f to generate Clr-f-specific mAb. Exclusive specificity of resulting $\mathrm{mAbs}$ $10 \mathrm{~A} 6$ and 10A8 for Clr-f was shown by flow cytometry using $\mathrm{CHO}$ cells ectopically expressing $\mathrm{Clr}-\mathrm{f}(\mathrm{CHO}-\mathrm{Clr}-\mathrm{f})$ as compared with other $\mathrm{Clr}$ molecules and by staining of sClr-fcoated microspheres (Figure 3a; Supplementary Figure S4). To assess endogenous expression of Clr- $\mathrm{f}$ by IECs, freshly isolated intestinal cells were stained with mAb $10 \mathrm{~A} 6$ and analyzed by flow cytometry. 10A6 markedly bound to IECs, but not to EpCAM-negative cells including IELs, and binding to IECs was specifically blocked by sClr-f (Figure $3 \mathbf{b}$ ). Of note, IECs did not uniformly express $\mathrm{Clr}-\mathrm{f}$ as there was a significant subset of IECs with an absent or low expression of Clr-f (Figure 3b). To allocate Clr-f-expressing cells within the intestinal epithelium, sections of the small intestine were stained with mAb 10A6. Notably, differentiated IECs lining the intestinal villi prominently and homogenously expressed Clr-f, whereas crypt cells did not express Clr-f (Figure $3 \mathrm{c}$ and $\mathbf{d}$; Supplementary Figure S5). Clr-f expression was also noted for the colon epithelium well in line with the presence of $\mathrm{Clr}-\mathrm{f}$ transcripts (Figure 3e).

Nkrp1g is a receptor for Clr-f expressed by a subset of IELs Distinct expression of Clr-f by IECs was intriguing and raised immediate questions regarding the immunological significance thereof. Recently, it has been reported that Clr-f is a ligand of Nkrp1g, a member of the mouse family of NKRP1 receptors. ${ }^{13,15}$ Of note, the genes of $\mathrm{Clr}-\mathrm{f}(\mathrm{Clec} 2 h)$ and Nkrplg $($ Klrblg) are located vis-à-vis in the NKC in a tail-to-tail orientation, following the principle of genetic linkage of NKRP1/CLEC2 receptor/ligand pairs ${ }^{11}$ (Figure 4a). In contrast to other members of the mouse NKRP1 family, physiologic expression of Nkrplg remained undefined, mainly due to the lack of Nkrp1g-specific mAb. ${ }^{23}$ Hence, we generated Nkrp1gspecific $\mathrm{mAb}$ by immunizing rats with the soluble CTLD of Nkrp1g (sNkrp1g) expressed in $293 \mathrm{~T}$ cells. The resulting $\mathrm{mAb}$ $8 \mathrm{~A} 10$ and $4 \mathrm{~A} 2$ specifically bound to NK-92MI cells ectopically expressing Nkrp1g, but did not cross-react with other NKRP1 receptors (Figure $4 \mathbf{b}$ and Supplementary Figure S6). We assayed an interaction of Nkrplg expressed in the context of NK-92MI cells with Clr-f using BWZ.36 reporter cells ${ }^{24}$

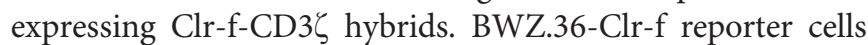


a

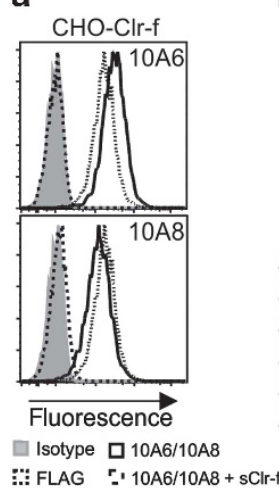

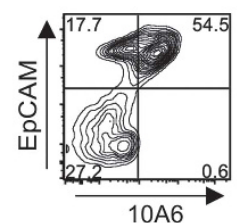

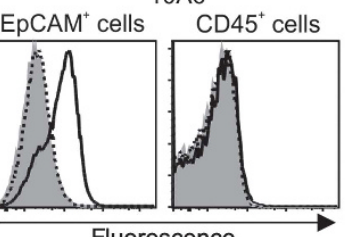

Fluorescence $\square$ Isotype $\square 10 \mathrm{~A} 6 \quad \mathbf{-} \cdot 10 \mathrm{~A} 6+\mathrm{sCl}-\mathrm{f}$
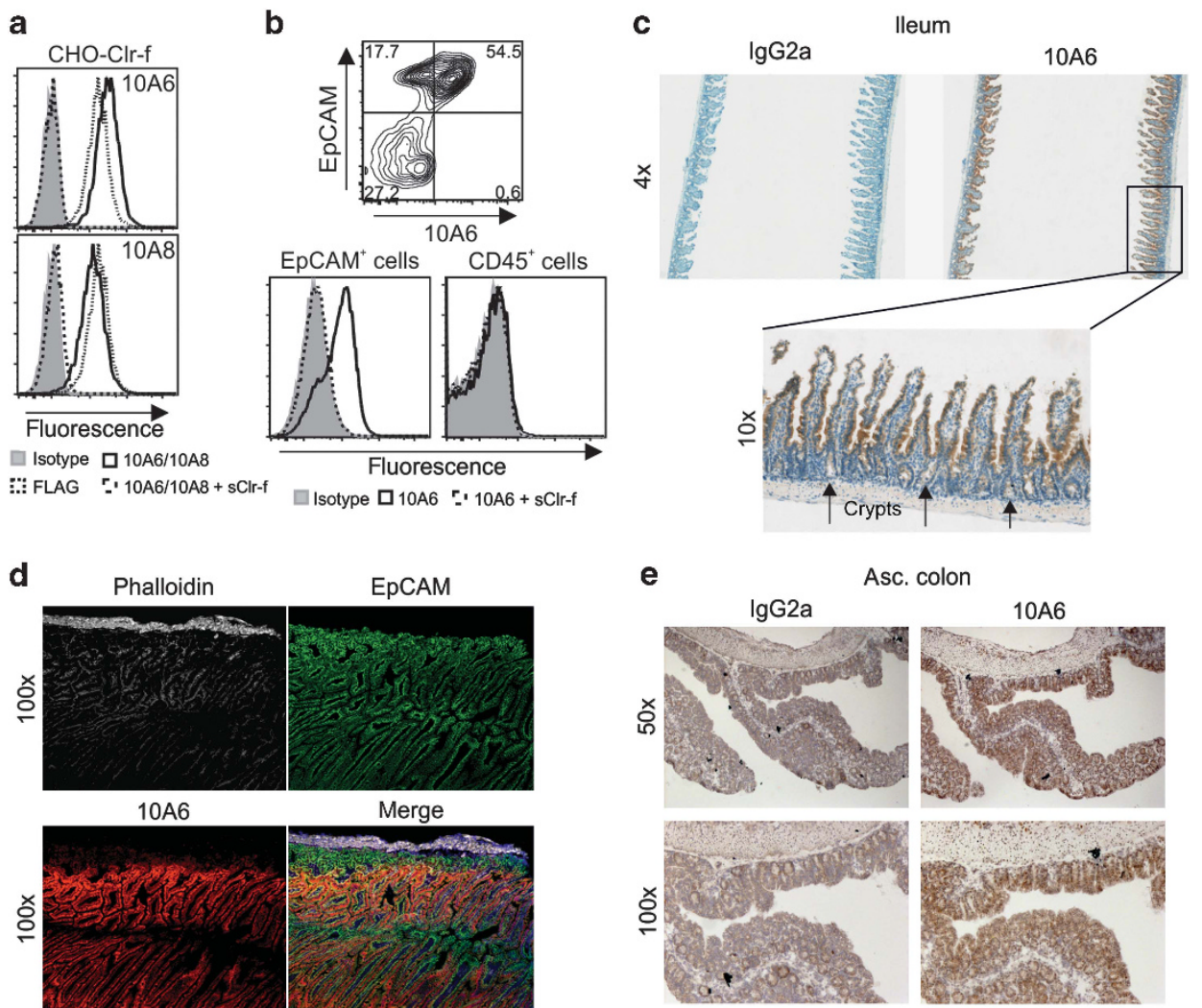

Figure 3 Clr-f expression on differentiated intestinal epithelial cells. (a) MAb 10A6 and 10A8 specifically bind to Clr-f on CHO-Clr-f cells (solid lines) comparable to FLAG-tag mAb M2 (dotted), but not when pretreated with sClr-f (dashed). (b) MAb $10 A 6$ detects $\mathrm{Clr}_{\text {-f }}$ on freshly isolated EpCAM ${ }^{+}$IEC, but not when pretreated with sClr-f (dashed line) and not on $\mathrm{CD} 45^{+}$IEL (solid line). (a and b) Isotype stainings are shaded. (c and d) Frozen sections of mouse C57BL/6 small intestine stained with (c) mAb 10A6 (right) and Mayer's hematoxylin or (d) with phalloidin (white), anti-EpCAM (green) and mAb 10A6 (red), respectively. Merged pictures include DAPI stainings (blue). (e) Frozen sections of mouse C57BL/6 ascending colon stained with mAb 10A6 (right) and Mayer's hematoxylin. (c and e) IgG2a served as a control (left). Asc. colon, ascending colon; Clr, C-type lectin-related; DAPI, 4,6-diamidino-2phenylindole; IEC, intestinal epithelial cell; IEL, intraepithelial lymphocyte.

strongly responded to cocultured NK-92MI-Nkrp1g cells (Figure 4c). Responses of the reporters could largely be blocked by the addition of anti-Clr-f mAb 10A8 or anti-Nkrp1g $\mathrm{mAb} 8 \mathrm{~A} 10$, respectively (Figure 4c). Further, there was also a pronounced binding of sClr-f tetramers to NK-92MI-Nkrp1g cells that was completely blocked by the addition of mAb $8 \mathrm{~A} 10$ (Figure 4d) and that was irrespective of sClr-f glycosylation (Supplementary Figure S7). Collectively, these results not only corroborated Nkrplg/Clr-f interaction previously shown by using Nkrp1g-CD3 $\zeta$ reporter cells, ${ }^{13,15}$ but also qualified $\mathrm{mAb}$ $10 \mathrm{~A} 8$ and $8 \mathrm{~A} 10$ as efficient blocking reagents of this interaction for further functional studies.

\section{Subsets of intestinal epithelial $\gamma \delta$ T cells and $\alpha \beta \mathrm{T}$ cells express Nkrp1g}

To determine and localize the cells expressing Nkrp1g, various tissues of C57BL/6 mice were screened by qPCR. Nkrp1g transcripts were most abundant in the spleen and small intestine, and also present in colon and other hematopoietic organs (Figure 5a). Further dissection of intestinal Nkrplg expression revealed a predominance of Nkrplg transcripts in
IELs ( $\sim 80$-fold of small intestine) whereas these were not detectable in IECs (Figure 5b), constituting an intestinal epithelial expression pattern obverse to that of Clr-f. Expression of Nkrp1g on IELs was further studied using mAb 8A10. Nkrp1g expression was detectable on a subset of $\mathrm{CD} 3{ }^{+} \mathrm{CD} 103^{+}$ IELs isolated from BALB/c mice and included both $\gamma \delta \mathrm{TCR}^{+}$ and $\alpha \beta \mathrm{TCR}^{+}$IELs (Figure 5c). Specificity of staining was verified by blockade with sNkrp1g (Figure 5c). Comprehensive analyses of IELs from $\mathrm{BALB} / \mathrm{c}$ and $\mathrm{C} 57 \mathrm{BL} / 6$ mice revealed higher frequencies of Nkrplg ${ }^{+}$IELs in BALB/c mice as compared with C57BL/6 mice (Figure 5d). Furthermore, frequencies of $\mathrm{Nkrp}^{+}{ }^{+}$IELs were quite variable in individual mice ranging from 0.5 to $13 \%$ for $\gamma \delta$ T cells and up to $2 \%$ for $\alpha \beta \mathrm{T}$ cells (Figure 5e). Frequencies of $\mathrm{Nkrp}^{+}{ }^{+} \gamma \delta$ IELs significantly increased in older mice, whereas the overall frequency of Nkrplg ${ }^{+}$IELs did not change (Figure 5f). The latter is likely due to an overall reduction of $\gamma \delta$ IELs (Supplementary Figure S8) caused by a constant expansion of the so-called "induced $\alpha \beta$ IELs". ${ }^{7}$ To allocate these Nkrp1g ${ }^{+}$ intestinal lymphocytes, sections of the small intestine were stained with anti-Nkrp1g mAb $4 \mathrm{~A} 2$ or $8 \mathrm{~A} 10$, respectively, 


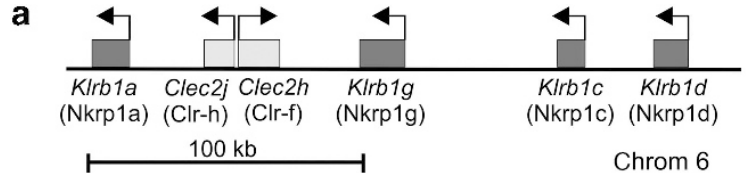

b

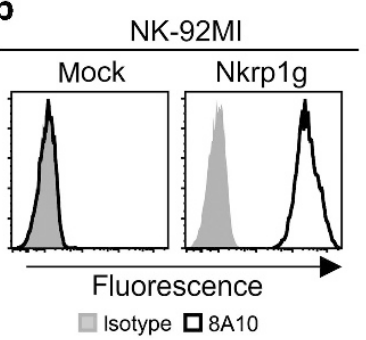

c

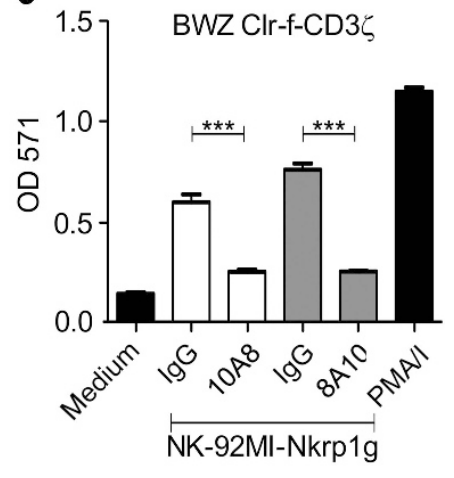

d NK-92Ml-Nkrp1g

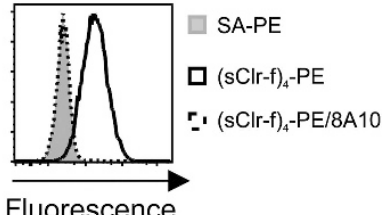

NK-92Ml-Nkrp1g

Figure 4 Clr-fligates Nkrp1g. (a) Genes of Clr-f (Clec2h) and its receptor Nkrp1g (KIrb1g) are tightly linked in the NKC. Boxes indicate genes, arrows transcriptional orientation. (b) MAb $8 \mathrm{~A} 10$ specifically binds to NK92MI-Nkrp1g cells, but not to control transductants (solid line). Isotype staining shaded. (c) BWZ.36 reporter cells expressing FLAG-tagged Clr-fCD3 $\zeta$ chimera respond to cocultured NK-92MI-Nkrp1g strictly depending on Nkrp1 $/ \mathrm{Cl}$-f interaction. Either reporter cells were pretreated with antiClr-f (10A8) or isotype control (open bars) or NK-92MI cells with antiNkrp1g (8A10) antibodies or an isotype control (gray bars) before coculture. Addition of medium only or PMA/ionomycin to reporter cells served as negative and positive control, respectively. One-way analysis of variance Tukey's post hoc ( $\left.{ }^{* \star *} P<0.0001\right)$. (d) sClr-f tetramers (solid line), but not control reagent (shaded), bind Nkrp1g on NK-92MI-Nkrp1g cells unless Nkrp1g was blocked by preincubation with mAb 8A10 (dotted line). Clr, C-type lectin-related; NKC, natural killer gene complex; PMA, phorbol 12-myristate 13-acetate.

revealing Nkrp1g ${ }^{+}$cells in intimate contact with intestinal epithelial cells (Figure 5g).

\section{Nkrp1g ${ }^{+}$IELs exhibit a $\gamma \delta \mathrm{TCR}^{\text {bright }} \mathrm{Nkg2a}^{-}$phenotype}

To further characterize Nkrplg-expressing IELs, Nkrp1g ${ }^{+}$and Nkrp1g ${ }^{-} \gamma \delta$ T cells were FACSorted using the Nkrp1g-specific mAb 8A10 (Supplementary Figure S9a). Faithful distinction of these subsets by mAb $8 \mathrm{~A} 10$ was confirmed by qPCR analyses of the sorted cells (Figure 6a) that were also subjected to microarray-based differential transcriptional profiling (Supplementary Figure S9b). For gene products for which suitable antibodies were not available, results from microarray studies were validated by qPCR of independently sorted IELs pooled from various mice. Consistently, an about twofold increase of transcripts of the integrin subunit CD11d and the close Nkrp1g relative Nkrp1f was detected in $\mathrm{Nkrplg}^{+} \gamma \delta$ $\mathrm{T}$ cells, whereas the levels of the chemokine CCL3/MIP- $1 \alpha$ were twofold lower (Figure 6b). Nkrplg ${ }^{+} \gamma \delta \mathrm{T}$ cells also expressed transcripts for TGF- $\beta 1$ and TGF- $\beta 3$, as well as for the keratinocyte growth factor (Supplementary Figure S9d).

Differential expression of Nkg2a could be confirmed by flow cytometry, with Nkg2a preferentially expressed on Nkrp1g $\gamma \delta$ IELs (Figure 6c). Notably, Nkrplg ${ }^{+} \gamma \delta$ T cells are characterized by a strong expression of the $\gamma \delta$ TCR complex (Figure 6d and e).

\section{Potent inhibition of effector cell responses by Nkrp1g/Clr-f interaction}

Considering the contiguity of Nkrplg ${ }^{+}$IELs and Clr- $\mathrm{f}^{+}$IECs in the intestinal epithelium, we wondered about the functional implications of this tissue-specific Nkrp1g/Clr-f interaction. As functional consequences of Nkrp1g ligation have not yet been reported, we directly addressed Nkrplg function in cocultures of NK-92MI-Nkrp1g cells with CHO cells. NK-92MI-Nkrp1g cells readily degranulated when cocultured with $\mathrm{CHO}$ cells, whereas no degranulation was detectable in the presence of $\mathrm{CHO}$ cells ectopically expressing Clr-f (Figure 7a and $\mathbf{b}$ ). Degranulation towards $\mathrm{CHO}-\mathrm{Cl}$-f cells could be restored in the presence of anti-Clr-f $\mathrm{mAb} 10 \mathrm{~A} 8$, while addition of the nonblocking Clr-f mAb 10A6 had no effect (Figure 7a and b). Accordingly, $\mathrm{CHO}-\mathrm{Clr}-\mathrm{f}$ cells were resistant to cytolysis by NK-92MI-Nkrp1g cells. However, resistance to cytolysis was abolished in the presence of $\mathrm{mAb} 10 \mathrm{~A} 8$, resulting in a cytolysis comparable to control CHO cells (Figure 7c). Altogether, these data demonstrate that Nkrplg acts as a potent inhibitory receptor when engaged by its cognate ligand $\mathrm{Clr}-\mathrm{f}$.

\section{Enhanced Clr-f expression on IECs upon poly(I:C) treatment can be sensed via Nkrp1g}

On the basis of the findings reported in this study, one may hypothesize that $\mathrm{Clr}-\mathrm{f}$ expression on IECs dampens effector functions of $\mathrm{Nkrplg}^{+}$IELs and that alterations in Clr-f expression levels, e.g., in course of an infection or an inflammation, may serve to modulate the responsiveness of these IELs. To address a potential regulation of Clr-f expression during an inflammatory challenge, mice were intraperitoneally injected with poly(I:C) and subsequently analyzed for expression of Clr-f and Nkrp1g, respectively. Sixteen hours post injection, there was a significant increase in Clr-f expression by IECs detected in situ by immunostaining and ex vivo by flow cytometry (Figure 8a-c). Increased expression of Clr-f following poly(I:C) injection was paralleled by a lower frequency of Nkrplg ${ }^{+}$IELs and a decrease in the respective Nkrplg expression levels, possibly indicating ligand-induced downregulation of Nkrplg (Figure 8d and e). To establish functional recognition of Clr-f on IECs by Nkrplg, we used

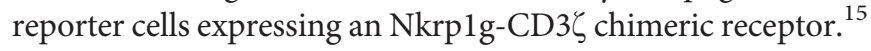
Human intestinal carcinoma cells ectopically expressing Clr-f (HCT116-Clr-f) were recognized by these reporter cells in a Clr-f-specific manner demonstrating that, in principle, Clr-f molecules on intestinal epithelial cells are amenable to Nkrplgmediated detection (Figure 8f and g). Finally, Nkrplg ${ }^{+}$ reporter cells were cocultured with IECs freshly isolated from phosphate-buffered saline-treated or poly(I:C)-treated mice. These reporter cells responded to IECs isolated from both groups of mice, but significantly stronger to IECs from poly(I:C)-treated mice, whereas responses were fully inhibited 


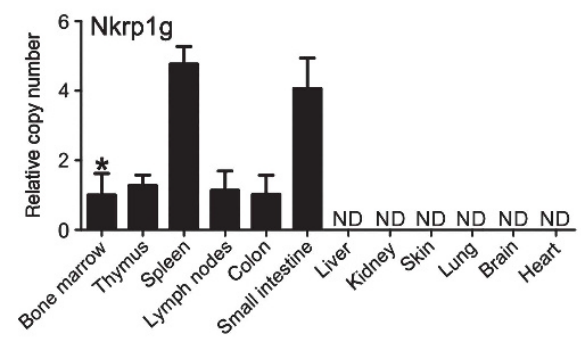

b

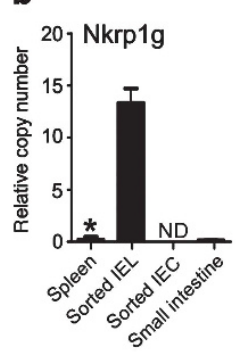

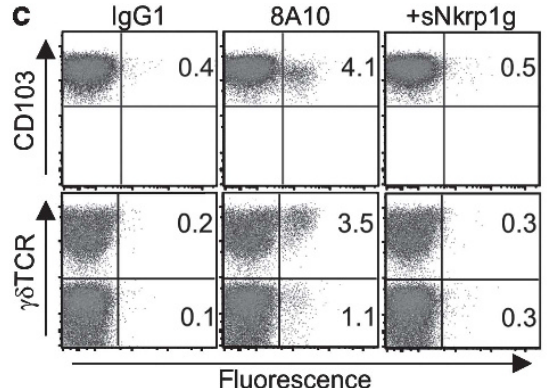

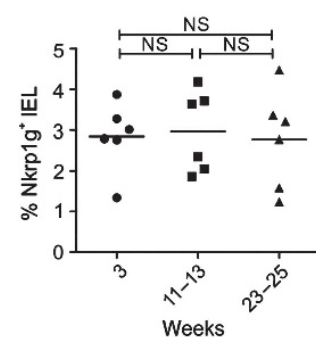

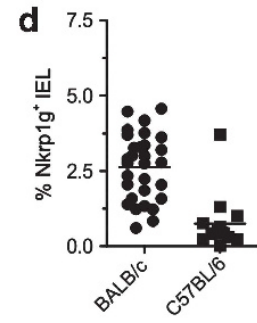

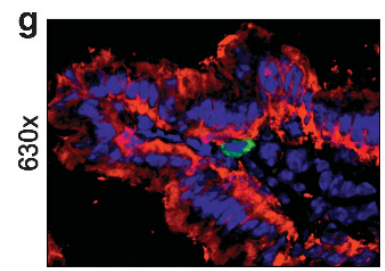

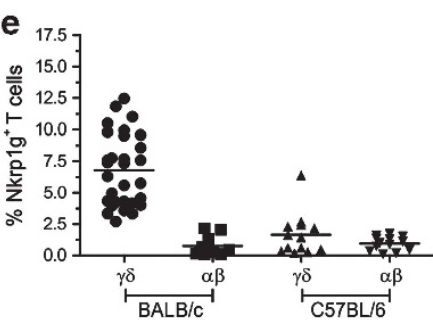

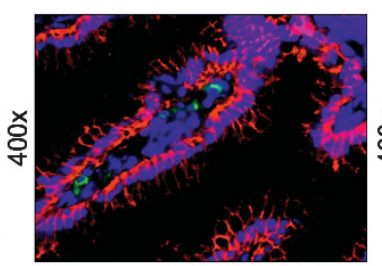

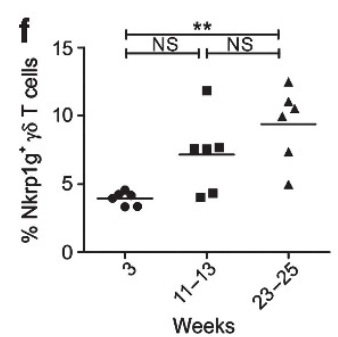

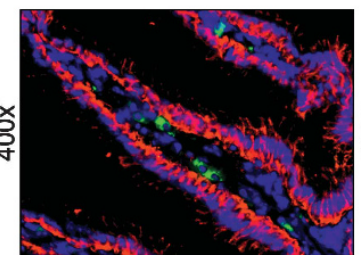

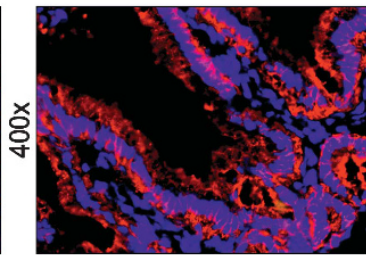

Figure 5 Nkrp1g is expressed on a subset of intraepithelial lymphocytes (a and $\mathbf{b})$ Relative abundance of Nkrp1g transcripts (a) in various tissues of female C57BL/6 mice and (b) FACSorted IEL and IEC. Tissues and cells of female C57BL/6 mice were analyzed by qPCR, levels of Nkrp1g transcripts were normalized to $18 \mathrm{~S}$ rRNA and arbitrarily set relative to tissues marked with an asterisk. (c) Intestinal IEL (gate: $\mathrm{CD} 103^{+}, \mathrm{CD}^{+}$) (upper) were assessed for expression of Nkrp1g (upper) or Nkrp1g co-expression with $\gamma \delta \mathrm{TCR}$ (lower) using mAb 8A10. Specificity of staining is shown by blockade with sNkrp1g (right); biotinylated rat IgG1 served as a control (left). (d and e) Compilation of frequencies of (d) $\mathrm{Nkrp}_{1 \mathrm{~g}}{ }^{+} \mathrm{CD} 3^{+} \mathrm{CD} 103^{+}$cells and of (e) Nkrp1 $\mathrm{g}^{+} \gamma \delta$ T cells and Nkrp1 ${ }^{+} \alpha \beta$ T cells among IEL from small intestines of BALB/c and C57BL/6 mice, respectively. (f) Frequencies of Nkrp1g ${ }^{+} \gamma \delta$ T cells (left) and $\mathrm{Nkrp} \mathrm{g}^{+} \mathrm{CD} 3^{+} \mathrm{CD}_{103^{+}}$cells (right) and among IEL from small intestines of differently aged BALB/c. Statistical differences in frequencies of $\mathrm{Nkrp} \mathrm{g}^{+}$cells are indicated by asterisks. One-way analysis of variance Tukey's post hoc (NS, not significant; $\left.{ }^{* \star} P<0.005\right)$. (g) Localization of Nkrp1g ${ }^{+}$IEL in situ. Frozen sections of mouse BALB/c small intestine stained for Nkrp1g (green) with mAb 4A2 (left and second left) or mAb 8A10 (second right), EpCAM (red), and counterstained with DAPI (blue). A representative isotype control staining (for mAb $8 A 10$ and $4 A 2$ ) is also shown (right). Clr, C-type lectin-related; DAPI, 4,6-diamidino-2-phenylindole; IEC, intestinal epithelial cell; IEL, intraepithelial lymphocyte; ND, not detectable; qPCR, quantitative polymerase chain reaction.

in the presence of the anti-Clr-f mAb $10 \mathrm{~A} 8$, demonstrating that they were Clr-f-specific (Figure $\mathbf{8 h}$ ). Altogether, these data show that upregulation of the intestinal epithelial Clr-f glycoproteins following poly(I:C)-exposure can be specifically sensed by Nkrp1g-expressing cells.

\section{DISCUSSION}

We here report the tissue-specific expression of the C-type lectin-like molecule Clr-f on differentiated mouse intestinal epithelial cells which contrasts the broad tissue expression of its close relative Clr-b, the best-studied member of the Clr family of CTLR. Although the original description of mouse Clr genes already provided first preliminary hints that their tissue expression may be differentially regulated, ${ }^{16}$ this was not elaborated by more in-depth studies until very recently. ${ }^{18,25}$ The subsequent characterization of certain Clr molecules as ligands of the genetically linked NKRP1 receptors fueled the interest in Clr family members, but for the most part, studies on
Clr expression remained limited to the levels of transcripts due to the lack of specific antibodies. ${ }^{18,25}$ Only for Clr-b, pronounced cell surface expression could be shown for various hematopoetic cells and for cell lines of various origins. ${ }^{12}$ The broad tissue expression of Clr-b and its interaction with the inhibitory Nkrp1d receptor on NK cells led to the hypothesis that the Nkrpld/Clr-b receptor/ligand pair may act as a second missing-self recognition system analogous to MHC class I. ${ }^{12,14,26,27}$ This idea was further supported by the observation that $\mathrm{Clr}$-b expression is reduced on many tumor cell lines and downregulated upon viral infection or cellular stress. ${ }^{12,14,21,26,27}$ In line with these previous studies, our tissue screen revealed a very broad and fairly uniform tissue expression of Clr-b except for the brain, where the non-NKC-encoded CLEC2 family member BACL is selectively expressed. ${ }^{28}$ Unlike Clr-b, Clr-g transcripts primarily locate to hematopoietic organs such as bone marrow and spleen, suggesting a preferential Clr-g expression by hematopoietic cells similar to the human CLEC2 


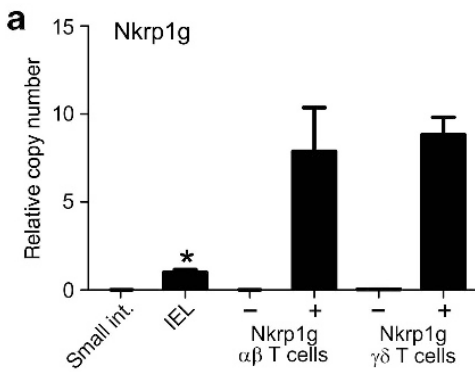

b
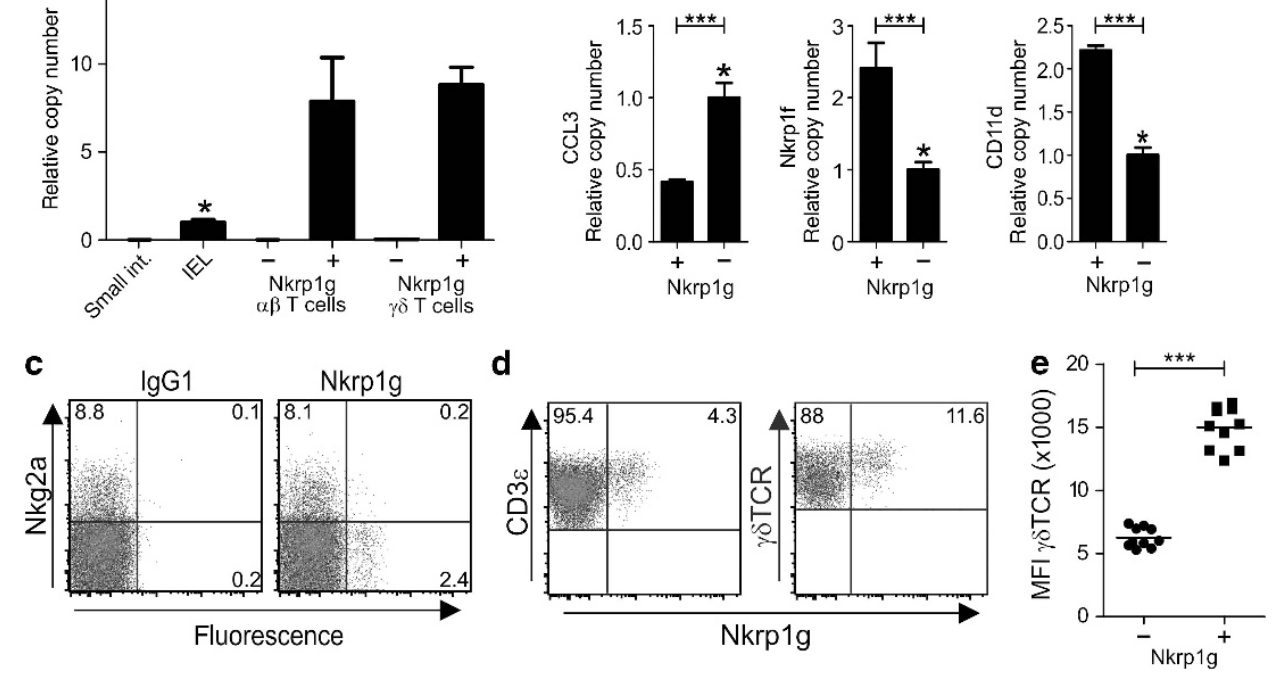

Figure 6 Characteristics of Nkrp1g ${ }^{+}$IEL. (a) Freshly isolated IEL from small intestines of BALB/c mice were FACSorted for Nkrp1g ${ }^{+}$or Nkrp1g ${ }^{-}$ subsets of $\alpha \beta$ T cells or $\gamma \delta$ T cells using mAb $8 A 10$ and purity of sorted cells verified by qPCR assessment of Nkrp1g expression. (b) Relative abundance of CCL3, Nkrp1f, and CD11d transcripts in FACSorted Nkrp1g ${ }^{+}$and Nkrp1g ${ }^{-} \gamma \delta$ IEL. (a and b) Levels of transcripts were normalized to 18S rRNA and set relative to samples marked with an asterisk. (c-e) IEL from C57BL/6 (c) and BALB/c mice (d) were analyzed for co-expression of Nkrp1g and (c) Nkg2a, (d) CD3 $\varepsilon$ or $\gamma \delta$ TCR, respectively. (e) Median fluorescence intensity (MFI) of $\gamma \delta$ TCR on Nkrp1 $\mathrm{g}^{-}$vs. Nkrp1g ${ }^{+} \gamma \delta$ T cells. Statistical differences in MFI of $\gamma \delta$ TCR are indicated by asterisks. T-test, unpaired, two-tailed $\left({ }^{* *} P<0.0005\right)$. IEL, intraepithelial lymphocyte; qPCR, quantitative polymerase chain reaction; small int., small intestine.

family members AICL and LLT1. ${ }^{11,17,29,30}$ The gut-associated expression pattern of Clr-f reported here was consistently observed independent of strain, sex, and age of mice and thus appears as a characteristic trait of the mouse intestinal epithelium. Apparently, Clr-f protein expression is abruptly acquired during IEC differentiation when leaving the crypts as only villous epithelial cells, but not crypt cells, express Clr-f molecules. The rather exclusive expression of $\mathrm{Clr}$-f by intestinal epithelial cells is reminiscent of the gut-restricted expression of the MHC class I-like molecule TL, a ligand of CD $8 \alpha \alpha$ homodimers characteristic of the so-called "natural" IELs. ${ }^{31,32}$ Recent studies showed that engagement of TL on IECs by CD $8 \alpha \alpha$ on IELs contributes to the generation of a pool of highaffinity effector memory CD $8 \alpha \beta$ IELs. ${ }^{33}$ Similarly, we show that IEC-specific expression of $\mathrm{Cl}-\mathrm{f}$ is matched by expression of its receptor Nkrp1g on a subset of IELs. These are "natural" IELs as they typically lack expression of CD5 (Supplementary Figure S9c) and include both intestinal $\alpha \beta$ and $\gamma \delta$ T cells, with a significantly higher representation among $\gamma \delta \mathrm{T}$ cells. We consistently observed lower frequencies of Nkrplg-expressing IELs in C57BL/6 mice as compared with $\mathrm{BALB} / \mathrm{c}$ mice indicating strain-specific genetic determinants of Nkrplg expression. Our current data also show increased frequencies of Nkrplg ${ }^{+}$ $\gamma \delta$ IEL among aged mice in line with an accumulation of senescent $\gamma \delta$ T cells. It was previously shown, that colonic $\gamma \delta$ $\mathrm{T}$ cells express proinflammatory cytokines and chemokines such as IL-1 $\beta$, CXCL1, CXCL2, and CXCL9 upon mucosal injury $^{34}$ and that these are also produced by $\gamma \delta$ IELs of the small intestine. ${ }^{35}$ We found Nkrplg ${ }^{+} \gamma \delta \mathrm{T}$ cells to express transcripts for TGF- $\beta 1$ and TGF- $\beta 3$, as well as for the keratinocyte growth factor and this may mediate both suppressive and regenerative effects on epithelial cells. Interestingly, expression of Nkg2a and Nkrp1g is discordant on IELs and may coincide with different effector functions. In humans, triggering of the inhibitory CTLR NKG2A on $\gamma \delta$ T cells has been shown to suppress cytotoxic $\alpha \beta$ T cells by the release of TGF- $\beta 1$ in patients with celiac disease. $^{9}$

Strangely enough, not only the genes of the receptor/ligand pair Nkrplg/Clr-f are opposed in tight genetic linkage in the NKC, but also the corresponding gene products are expressed by intimate, yet unrelated neighbors in the mouse intestinal epithelium (Figure 5g). Attribution of Nkrp1g and Clr-f expression to the intestinal epithelial compartment raises questions about the functional relevance thereof. Although direct assessment of the functional implications of the Nkrp1g/ Clr-f interaction in the gut epithelium must await the availability of genetically modified mice, our data clearly demonstrate that the Nkrplg/Clr-f interaction provides a strong inhibitory signal in line with the presence of a putative immunoreceptor tyrosine-based inhibitory motif in the cytoplasmic domain of Nkrp1g. As Clr-f molecules are expressed at the surface of intestinal epithelial cells and thus can be sensed by adjacent IELs via the inhibitory receptor Nkrplg, it is conceivable that $\gamma \delta$ T cells are capable to survey the "state" of intestinal epithelial cells by means of the Nkrplg/Clr-f interaction. As previously reported for $\mathrm{Clr}-\mathrm{b},{ }^{21,27}$ downregulation of Clr-f may indicate cellular stress, pathogenic challenge, or mucosal injury, and unleash effector responses of Nkrplg-expressing $\gamma \delta \mathrm{T}$ cells, such as the release of proinflammatory cytokines and chemokines. In light of the 
a

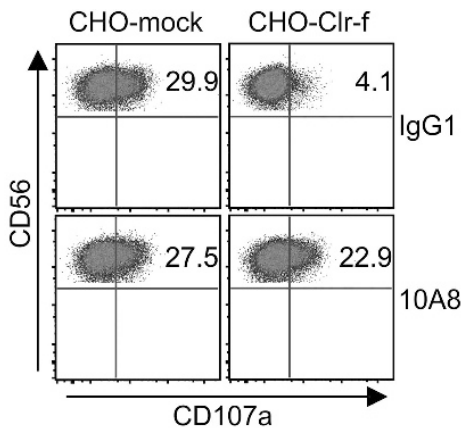

b
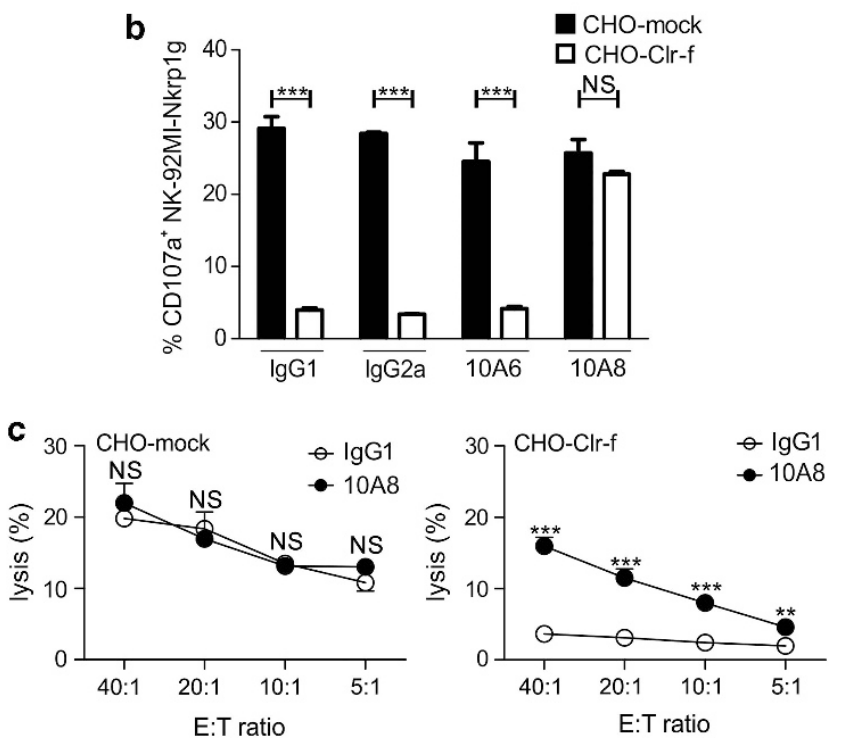

Figure 7 Nkrp1g potently inhibits effector functions upon Clr-f engagement. (a and $\mathbf{b}$ ) Degranulation of NK-92MI-Nkrp1g in cocultures with mock- or Clr-f-transfected $\mathrm{CHO}$ cells, in the presence of Clr-f-specific mAb 10A8 (blocking Nkrp1g/Clr-f interaction) or 10A6 (non-blocking) or isotype controls, respectively. (a) Representative dot blots and (b) compilation of experimental data. (c) Cytolysis of mock- or Clr-ftransfected $\mathrm{CHO}$ cells by NK-92MI-Nkrp1g cells in the presence of $\mathrm{mAb}$ $10 A 8$ or isotype control. (b and c) Errors bars of triplicates are shown and statistical significance of differences between respective data pairs is given (two-way analysis of variance Bonferroni post tests: ${ }^{* \star \star} P<0.001$; ${ }^{* \star} P<0.01$; NS, not significant). Clr, C-type lectin-related.

highly proliferative nature and demanding microbial exposure of the intestinal epithelium, such a dedicated immunosurveillance mechanism may aid in controlling the integrity of the epithelial barrier. Alternatively, Nkrplg may constrain the reactivity of IELs by the continuous engagement of Clr-f, and this mechanism may contribute to the control of IEL effector responses in an environment rich in immunostimulatory compounds and microbiota. First support for this latter idea was gained from experiments demonstrating that expression of Clr-f on IEC is substantially increased upon treatment with poly(I:C) and that this increase can be sensed via Nkrpglg. Such a mechanism may provide increased protection from IEL-mediated damage.

Taken together, we find Clr-f almost exclusively expressed by differentiated intestinal epithelial cells matched by a rather specific expression of Nkrplg on a subset of intestinal IELs. As engagement of Clr-f by Nkrplg strongly inhibits effector responses, we hypothesize that this cognate receptor/ligand pair may contribute to the dedicated immunosurveillance of the epithelial barrier of the mouse gut.

\section{METHODS}

Mice. C57BL/6 and BALB/c mice were purchased from Harlan Laboratories and housed at the local animal facility. 6- to 8-week-old $\mathrm{BALB} / \mathrm{c}$ mice were injected intraperitoneally with $30 \mu \mathrm{g}$ poly(I:C) per gram body weight (high molecular weight poly(I:C) from Invivogen, Toulouse, France). After $16 \mathrm{~h}$ mice were killed for isolation of IEC and IEL. Animal experiments were approved by the local authorities (Regierungspräsidium, Darmstadt, Germany; permit numbers F146/ 04 and F146/Anz03) and performed in full compliance with the respective national guidelines.

Quantitative PCR. RNA was isolated from cells using peqGOLD TriFast (Peqlab, Erlangen, Germany) or RNAqueous-Micro Kit (Life Technologies, Darmstadt, Germany) and converted into cDNA using M-MLV RT (H-) (Promega, Mannheim, Germany) according to the manufacturer's protocol after treatment with DNase I for $30 \mathrm{~min}$ at $37^{\circ} \mathrm{C}$. cDNA was amplified on the real-time PCR system StepOnePlus (Applied Biosystems, Foster City, CA) using SYBR Green chemistry (Roche, Mannheim, Germany) for detection (primer pairs are shown in Supplementary Table S1). Copy numbers were normalized with copy numbers of $18 \mathrm{~S}$ rRNA ( $\Delta \mathrm{Ct}$ method).

Transfectants. 293 and 293T cell lines were cultured in Dulbecco's Modified Eagle's medium. CHO, HCT116, and NK-92MI cell lines were cultured in Iscove's Modified Dulbecco's Medium. CHO, HCT116, and 293T cells were transfected using FuGENE HD (Roche) with cDNA of Clec2h, Clec2d, or Clec2i containing a carboxyterminal FLAG- and hexahistidine-tag in RSV.5neo expession vector. Stably transfected cells were selected in G418 containing medium $\left(2 \mathrm{~m} \mathrm{ml}^{-1}\right)$. NK-92MI were transduced with retroviruses generated by transfection of phoenix-ampho cells (Nolan Laboratory, Stanford, CA) with the vector pMXsIP kindly provided by Toshio Kitamura (University of Tokyo) ${ }^{36}$ containing the Nkrplg cDNA with a carboxyterminal FLAG-tag. Transduced NK-92MI cells were selected in puromycin-containing medium $\left(7 \mu \mathrm{g} \mathrm{ml}^{-1}\right)$.

Chimeric reporter construct. For the generation of reporter constructs, the nucleotide sequence for the CTLD of Clr-f was amplified with the following pairs of oligonucleotides: $5^{\prime}$-CAGCAACATGCC GGCCATGGAAGACCGTGAA- $3^{\prime}$ and $5^{\prime}$-CTCGAGCACATGCCTT GGTACACTTC-3' from mouse small intestine cDNA. These oligonucleotides contained overlapping sequences for fusion with sequences encoding for the stalk region and transmembrane domain of Ly49a and the cytoplasmic domain of mouse $\mathrm{CD} 3 \zeta$, amplified from an existing reporter construct (NKG2D-Ly49a-CD3ל). The resulting fusion products were equipped with SacII and XhoI restriction sites and cloned into an RSV.5neo expression vector containing C-terminal FLAG- and hexahistidine tags.

Reporter cell assay. BWZ.36 reporter cells, a kind gift of N. Shastri, University of California, Berkeley, ${ }^{24}$ were cultured in RPMI 1640. Transfection of BWZ.36 cells was performed by electroporation using $20 \mu \mathrm{g}$ of plasmid DNA and a Gene Pulser Xcell (Bio-Rad, Munich, Germany) ( $250 \mathrm{~V}, 950 \mu \mathrm{F})$ followed by selection with G418 containing medium $\left(1.8 \mathrm{mg} \mathrm{ml}^{-1}\right)$. BWZ.36 reporter cells $\left(1 \times 10^{5}\right.$ per well $)$ were cocultured overnight with $1 \times 10^{5}$ target cells in the presence of IgG, anti-Clr-f mAb 10A8, or anti-Nkrp1g mAb 8A10 $\left(10 \mu \mathrm{g} \mathrm{ml}^{-1}\right)$, respectively. Cells were lysed in the presence of chlorophenol red- $\beta$-Dgalactopyranoside (final concentration: $150 \mu \mathrm{m}$; Sigma, Steinheim, Germany) and $\beta$-galactosidase activity was determined by measuring absorbance at $571 \mathrm{~nm}$. For controls, BWZ.36 reporter cells were cultured in medium or stimulated with $25 \mathrm{ng}$ PMA (phorbol 12- 

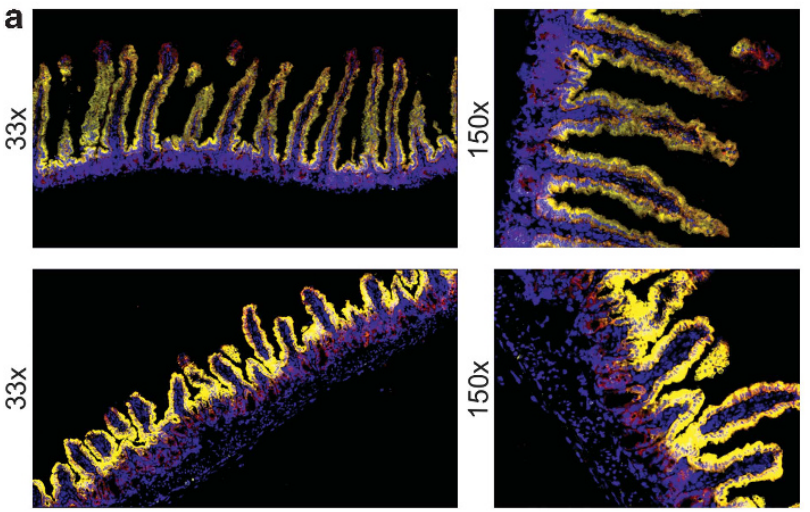

d

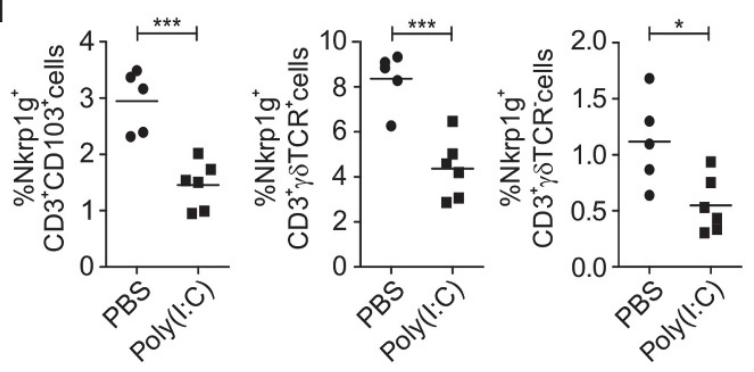

e

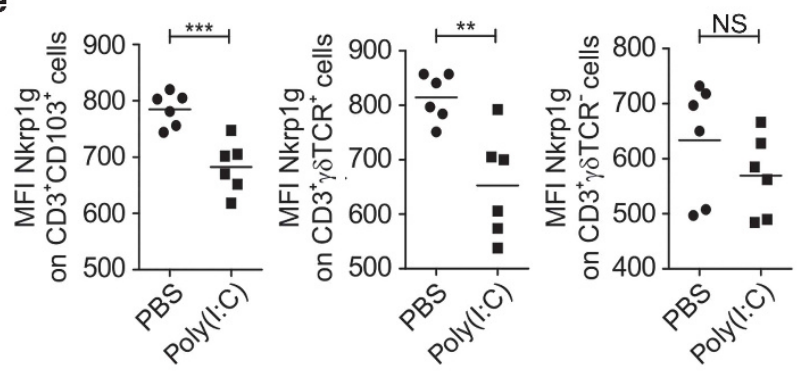

b

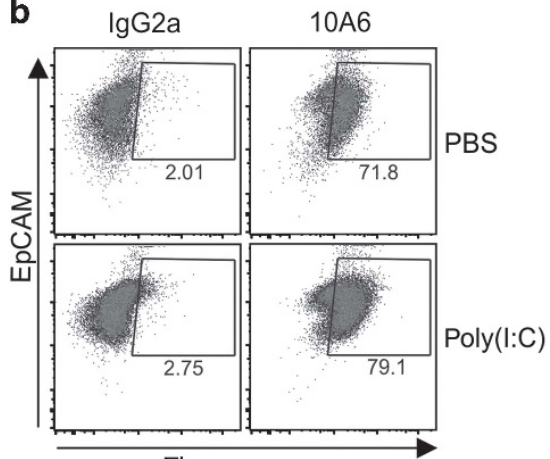

Fluorescence

f

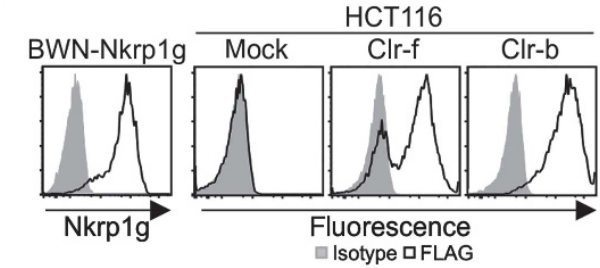

g

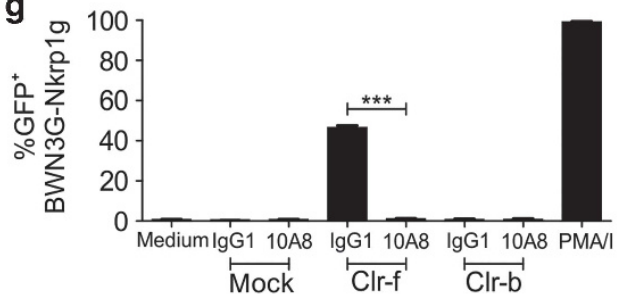

h

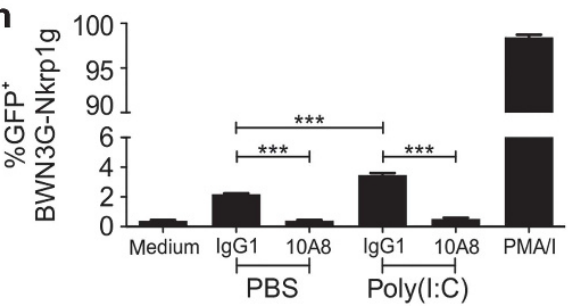

Figure 8 Upregulation of Clr-f molecules on IEC subsequent to poly $(\mathrm{I}: \mathrm{C})$ treatment can be sensed by Nkrp1g. (a) Frozen sections of the small intestine of $\mathrm{BALB} / \mathrm{c}$ mice treated with PBS (upper) or poly(l:C) (lower) stained with mAb $10 \mathrm{A6}$ (yellow) and anti-EpCAM (red) and counterstained with DAPI (blue). (b and c) Flow cytometric analyses of IEC from PBS- or poly(I:C) treated mice for Clr-f surface expression (mAb 10A6). Representative dot plots (b) and compiled data from six mice per group (c). (d and e) Flow cytometric analyses of IEL from PBS- or poly(l:C) treated mice for Nkrp1g expression (mAb 8A10). Compiled data (six mice per group) for (d) frequencies of Nkrp1g ${ }^{+}$cells or (e) MFI of Nkrp1g expression levels on Nkrp1g ${ }^{+}$cells. (f) Nkrp1g-CD3C expression of BWN3G reporter cells assessed with mAb 8A10 (left panel). HCT116 cells stably transfected with FLAG-tagged Clr-f or Clr-b, or mocktransfected cells stained with FLAG-tag mAb M2 (solid lines). Isotype control stainings are shaded. ( $\mathbf{g}$ and $\mathbf{h}$ ) Functional recognition of Clr-f (g) on HCT116-Clr-f cells or (h) on freshly isolated IEC from PBS- or poly(l:C)-treated BALB/c mice by cocultured BWN3G-Nkrp1g reporter cells. Specific recognition of $\mathrm{Clr}$-f was abrogated by addition of anti-Clr-f mAb 10A8 to the cocultures. Negative (medium) and positive controls (PMA/ionomycin) are also shown. One-way analysis of variance Tukey's post hoc $\left(^{* * \star} \mathrm{P}<0.0001\right)$. Clr, C-type lectin-related; DAPI, 4,6-diamidino-2-phenylindole; IEC, intestinal epithelial cell; IEL, intraepithelial lymphocyte; MFI, median fluorescence intensity; NS, not significant, PBS, phosphate-buffered saline; PMA, phorbol 12myristate 13-acetate.

myristate 13 -acetate) and $1 \mu \mathrm{M}$ ionomycin. BWN3G reporter cells expressing chimeric Nkrplg-CD3 $\zeta$ molecules and assay conditions were described previously. ${ }^{15}$ A total of $5 \times 10^{4}$ BWN3G reporter cells were cocultured with $1 \times 10^{5}$ HCT116 or $1.25 \times 10^{5}$ IECs, respectively. Stimulator cells and reporter cells were incubated overnight in the presence of $10 \mu \mathrm{g} \mathrm{ml}^{-1}$ rat IgG1 or anti-Clr-f mAb 10A8. Reporter cells were analyzed for induction of EGFP expression by flow cytometry.

Isolation of IECs and IELs. IECs and IELs were purified with modifications as described previously. ${ }^{37}$ Briefly, after removal of Peyer's patches, sections of the small intestines were washed with ice- cold phosphate-buffered saline, incubated at $37^{\circ} \mathrm{C}$ in Hank's Balanced Salt Solution containing $1 \mathrm{~mm}$ EDTA and $1 \mathrm{~mm}$ DTT for $45 \mathrm{~min}$ and vortexed before enrichment of lymphocytes by Percoll gradient centrifugation (GE Healthcare, Munich, Germany). Small intestines of 6- to 12 -week-old mice were used unless stated otherwise.

Recombinant proteins and antibodies. Soluble recombinant ectodomains of Nkrp1g (sNkrp1g: Pro66 through Val214) and Clr-f (sClr-f: Pro78 through Val218) were purified from supernatants of 293 cells stably transfected with the respective cDNA containing BirA-, c-myc-, and hexahistidine tags as previously reported for rNKp80. ${ }^{29}$ For gel filtration analysis, sCTLR were loaded onto a HiLoad 16/60 Superdex 
200 gel filtration column at a flow rate of $1 \mathrm{ml} \mathrm{min}^{-1}$. Ectodomains were biotinylated using BirA ligase (Avidity, Aurora, CO) and, before use, either immobilized on streptavidin-coated microspheres (Bangs Laboratories, Fishers, IN) or tetramerized using fluorochrome-labeled streptavidin (Jackson ImmunoResearch, Newmarket, UK). Clr-fspecific mAb 10A6 and 10A8 were generated by immunizing LOU/C rats (Harlan, Boxmeer, The Netherlands) with sClr-f and resulting hybridoma screened for specific reactivity against $\mathrm{CHO}-\mathrm{Cl}-\mathrm{f}$ transfectants. Nkrp1g-specific mAb 8A10 and 4A2 were generated by immunizing $\mathrm{CD}$ rats (Charles River, Köln, Germany) with sNkrplg and resulting hybridoma screened for specific reactivity against NK-92MI-Nkrp1g transductants.

Cytotoxicity, degranulation. $\mathrm{CHO}$ transfectants (targets, $\mathrm{T}$ ) were labeled with ${ }^{51} \mathrm{Cr}$ for $2 \mathrm{~h}$ at $37{ }^{\circ} \mathrm{C}$ and cocultured with NK-92MI transductants (effectors, E) for $4 \mathrm{~h}$ at $37{ }^{\circ} \mathrm{C}$ at different E:T ratios. Supernatant were mixed with OptiPhase Supermix scintillation cocktail (PerkinElmer, Waltham, MA) in an IsoPlate-96 and measured with a MicroBeta ${ }^{2}$ plate counter (PerkinElmer). In blocking experiments, antibodies (anti-Clr-f mAb 10A8, isotype) were used at a final concentration of $10 \mu \mathrm{g} \mathrm{ml}^{-1}$. Percent-specific lysis was calculated as follows: $100 \times$ (experimental release - spontaneous release)/ (maximum release - spontaneous release). Degranulation of NK92MI transductants was quantified by analysis of surface CD107a after $1 \mathrm{~h}$ coculture with $\mathrm{CHO}$ transfectants in the presence of mAb $10 \mathrm{~A} 8$ or an isotype control $\left(10 \mu \mathrm{g} \mathrm{ml}^{-1}\right)$.

Immunohistology. Immunohistochemistry was performed on cryosections of small intestine samples from C57BL/6 mice. Sections were fixed in cold acetone for $10 \mathrm{~min}$ and subsequently dried at room temperature. Specimens were stained with the MaxHetero Rat on Mouse Polymer HRP Detection Kit (MaxVision Bioscience, Bothell, WA) following manufacturers' protocol with anti-Clr-f mAb 10A6 or respective isotype control as primary $\mathrm{mAbs}$. Finally, cryosections were developed with $\mathrm{DAB}\left(3,3^{\prime}\right.$-diaminobenzidine) and counterstained with Mayer's hematoxylin. For immunostaining, cryosections of mouse intestinal samples were fixed with acetone. Slides were air dried and rehydrated before blocking in $10 \%$ donkey or goat serum. Sections were incubated in the presence of the anti-Clr-f antibody (mAb 10A6) or hybridoma supernatant containing anti-Nkrp1g mAb $8 \mathrm{~A} 10$ or $4 \mathrm{~A} 2$ for $1 \mathrm{~h}$ at room temperature or the respective isotype control. Secondary antibody staining was performed using a Cy3-conjugated donkey anti-rat antibody (Jackson ImmunoResearch) or Cy3conjugated goat anti-rat antibody (BioLegend, London, UK) for $0.5-1 \mathrm{~h}$ at room temperature. After washing, sections were stained with an APC-conjugated EpCAM antibody (eBioscience, Frankfurt, Germany) and fluoroscein isothiocyanate-conjugated phalloidin (Sigma) or with an Alexa647-conjugated EpCAM antibody (BioLegend). The specificity of the primary antibody was confirmed by preincubation for $1 \mathrm{~h}$ at room temperature in the presence or absence of $10 \mu \mathrm{g} \mathrm{ml}^{-1}$ of $\mathrm{sClr}-\mathrm{f}$. Slides were mounted in 4,6-diamidino-2phenylindole-containing Vectashield (Biozol, Eching, Germany). Tissue sections were visualized using an ApoTome-equipped Axioplan 2 microscope connected to an AxioCam Mr digital Camera (Carl Zeiss MicroImaging, Jena, Germany) or a DMI 6000B microscope connected to a DFC3000 camera (Leica Microsystems, Heerbrugg, Switzerland).

Immunoblotting. For immunoblot analysis, transiently transfected $293 \mathrm{~T}$ cells were lysed using lysis buffer $(50 \mathrm{~mm}$ Tris $\mathrm{pH} 8,150 \mathrm{~mm} \mathrm{NaCl}$, $1 \%$ NP-40) containing the complete protease inhibitor cocktail (Roche). For deglycosylation, samples were treated with PNGase-F (New England Biolabs, Frankfurt, Germany) according to manufacturer's instructions for $1 \mathrm{~h}$ at $37^{\circ} \mathrm{C}$. Thirty micrograms of total lysate were separated by SDS-polyacrylamide gel electrophoresis in a Mini-PROTEAN Tetra Cell (Bio-Rad) and transferred to nitrocellulose membranes (GE Healthcare) by semi-dry blotting. Membranes were stained with FLAG-tag mAb M2 (Sigma) in a final concentration of $1 \mu \mathrm{g} \mathrm{ml}^{-1}$ followed by detection using a horseradish peroxidase-conjugated goat anti-mouse IgG antibody (Jackson ImmunoResearch).

Flow cytometry and cell sorting. For analysis of cell surface expression, cells were washed with ice-cold FACS (fluorescenceactivated cell sorting) buffer (phosphate-buffered saline, $2 \%$ fetal calf serum, $2 \mathrm{~mm}$ EDTA, $0.01 \%$ sodium azide) and $\mathrm{Fc}$ receptors were blocked with $30 \mu \mathrm{g} \mathrm{ml}^{-1}$ mouse IgG (Sigma) for $20 \mathrm{~min}$ at $4{ }^{\circ} \mathrm{C}$. Cells were then stained with relevant antibodies for $20 \mathrm{~min}$ at $4{ }^{\circ} \mathrm{C}$ and washed again after each staining step with FACS buffer. Soluble $\mathrm{Clr}$-f-coated microspheres were also stained for $20 \mathrm{~min}$ at $4{ }^{\circ} \mathrm{C}$. Flow cytometry analysis was performed with a FACS Canto II (BD Biosciences, Heidelberg, Germany) and data analyzed using FlowJo (Tree Star, Ashland, OH). Antibodies used: anti-CD3e-PerCP, antiCD103-PE, anti- $\gamma \delta$ TCR-PE (all from BioLegend), anti-human CD107a-APC, anti-human CD56-PE (all from BD Biosciences), FLAG-tag mAb M2 (Sigma), anti-Nkrp1g mAb 8A10, and anti-Clr-f $\mathrm{mAb} 10 \mathrm{~A} 6$. Unconjugated antibodies were stained with Alexa647conjugated $\mathrm{F}(\mathrm{ab})_{2}$-fragments of mouse anti-rat IgG (Jackson ImmunoResearch). Single-cell suspensions of intraepithelial lymphocytes were sorted (purity $>98 \%$ ) using a BD FACSAria III cell sorter (BD Biosciences).

Statistical analysis. Statistical analyses as detailed in the figure legends were performed with GraphPad Prism 5 (GraphPad Software, San Diego, CA).

SUPPLEMENTARY MATERIAL is linked to the online version of the paper at http://www.nature.com/mi

\section{ACKNOWLEDGMENTS}

We thank Praveen Mathoor and Sandra Tafferner for excellent technical assistance, and Andreas Heumüller for experimental help. This work was supported by grants from the German Research Council to A.S. (STE 828/ 5-1; STE 828/5-2) and to M.W.H. (SFB 900/A4; HO 2236/8-1).

\section{DISCLOSURE}

The authors declared no conflict of interest.

(c) 2015 Society for Mucosal Immunology

\section{REFERENCES}

1. Duerr, C.U. \& Hornef, M.W. The mammalian intestinal epithelium as integral player in the establishment and maintenance of host-microbial homeostasis. Semin. Immunol. 24, 25-35 (2012).

2. Gallo, R.L. \& Hooper, L.V. Epithelial antimicrobial defence of the skin and intestine. Nat. Rev. Immunol. 12, 503-516 (2012).

3. Witherden, D.A. \& Havran, W.L. Cross-talk between intraepithelial gammadelta T cells and epithelial cells. J. Leukoc. Biol. 94, 69-76 (2013).

4. Neish, A.S. The gut microflora and intestinal epithelial cells: a continuing dialogue. Microbes Infect. 4, 309-317 (2002).

5. Hooper, L.V. \& Macpherson, A.J. Immune adaptations that maintain homeostasis with the intestinal microbiota. Nat. Rev. Immunol. 10, 159-169 (2010).

6. Maloy, K.J. \& Powrie, F. Intestinal homeostasis and its breakdown in inflammatory bowel disease. Nature 474, 298-306 (2011).

7. Cheroutre, H. et al. The light and dark sides of intestinal intraepithelial lymphocytes. Nat. Rev. Immunol. 11, 445-456 (2011).

8. Yokoyama, W.M. \& Plougastel, B.F. Immune functions encoded by the natural killer gene complex. Nat. Rev. Immunol. 3, 304-316 (2003).

9. Bhagat, G. et al. Small intestinal CD8+TCRgammadelta + NKG2A + intraepithelial lymphocytes have attributes of regulatory cells in patients with celiac disease. J. Clin. Invest. 118, 281-293 (2008).

10. Van Den Broeck, T. et al. Differential Ly49e expression pathways in resting versus TCR-activated intraepithelial gammadelta Tcells. J. Immunol. 190, 1982-1990 (2013) 
11. Vogler, I. \& Steinle, A. Vis-a-vis in the NKC: genetically linked natural killer cell receptor/ligand pairs in the natural killer gene complex (NKC). J. Innate Immun. 3, 227-235 (2011).

12. Carlyle, J.R. et al. Missing self-recognition of Ocil/Clr-b by inhibitory NKRP1 natural killer cell receptors. Proc. Natl. Acad. Sci. USA 101, 3527-3532 (2004).

13. Chen, P. et al. Analysis of the mouse 129-strain Nkrp1-Clr gene cluster reveals conservation of genomic organization and functional receptorligand interactions despite significant allelic polymorphism. Immunogenetics 63, 627-640 (2011).

14. lizuka, K. et al. Genetically linked C-type lectin-related ligands for the NKRP1 family of natural killer cell receptors. Nat. Immunol. 4, 801-807 (2003).

15. Kveberg, L. et al. Phylogenetic and functional conservation of the NKR-P1F and NKR-P1 G receptors in rat and mouse. Immunogenetics 63, 429-436 (2011).

16. Plougastel, B. et al. Cloning of Clr, a new family of lectin-like genes localized between mouse Nkrp1a and Cd69. Immunogenetics 53, 209-214 (2001).

17. Tian, W. et al. C-type lectin OCILRP2/Clr-g and its ligand NKRP1f costimulate T cell proliferation and IL-2 production. Cell. Immunol. 234, 39-53 (2005).

18. Zhang, Q. et al. Mouse Nkrp1-Clr gene cluster sequence and expression analyses reveal conservation of tissue-specific MHC-independent immunosurveillance. PLoS One 7, e50561 (2012).

19. Spreu, J. et al. CLEC2A: a novel, alternatively spliced and skin-associated member of the NKC-encoded AICL-CD69-LLT1 family. Immunogenetics 59, 903-912 (2007).

20. Spreu, J. et al. Interaction of C-type lectin-like receptors NKp65 and KACL facilitates dedicated immune recognition of human keratinocytes. Proc. Natl. Acad. Sci. USA 107, 5100-5105 (2010).

21. Fine, J.H. et al. Chemotherapy-induced genotoxic stress promotes sensitivity to natural killer cell cytotoxicity by enabling missing-self recognition. Cancer Res. 70, 7102-7113 (2010).

22. Williams, K.J. et al. Poxvirus infection-associated downregulation of C-type lectin-related-b prevents NK cell inhibition by NK receptor protein1B. J. Immunol. 188, 4980-4991 (2012).

23. Aust, J.G. et al. The expression and function of the NKRP1 receptor family in C57BL/6 mice. J. Immunol. 183, 106-116 (2009).
24. Sanderson, S. \& Shastri, N. LacZ inducible, antigen/MHC-specific T cell hybrids. Int. Immunol. 6, 369-376 (1994).

25. Flornes, L.M. et al. The complete inventory of receptors encoded by the rat natural killer cell gene complex. Immunogenetics 62, 521-530 (2010).

26. Plougastel, B.F. \& Yokoyama, W.M. Extending missing-self? Functional interactions between lectin-like NKrp1 receptors on NK cells with lectin-like ligands. Curr. Top. Microbiol. Immunol. 298, 77-89 (2006).

27. Voigt, S. et al. Cytomegalovirus evasion of innate immunity by subversion of the NKR-P1B:Clr-b missing-self axis. Immunity 26, 617-627 (2007).

28. Lysenko, O. et al. BACL is a novel brain-associated, non-NKC-encoded mammalian C-type lectin-like receptor of the CLEC2 family. PLoS One 8, e65345 (2013).

29. Welte, S. et al. Mutual activation of natural killer cells and monocytes mediated by NKp80-AICL interaction. Nat. Immunol. 7, 1334-1342 (2006).

30. Germain, C. et al. Induction of lectin-like transcript 1 (LLT1) protein cell surface expression by pathogens and interferon-gamma contributes to modulate immune responses. J. Biol. Chem. 286, 37964-37975 (2011).

31. Liu, Y. et al. The crystal structure of a TL/CD8alphaalpha complex at 2.1 A resolution: implications for modulation of $\mathrm{T}$ cell activation and memory. Immunity 18, 205-215 (2003).

32. Leishman, A.J. et al. T cell responses modulated through interaction between CD8alphaalpha and the nonclassical MHC class I molecule, TL. Science 294, 1936-1939 (2001).

33. Huang, Y. et al. Mucosal memory CD8(+) T cells are selected in the periphery by an MHC class I molecule. Nat. Immunol. 12, 1086-1095 (2011).

34. Ismail, A.S. et al. Reciprocal interactions between commensal bacteria and gamma delta intraepithelial lymphocytes during mucosal injury. J. Immunol. 182, 3047-3054 (2009).

35. Ismail, A.S. et al. Gammadelta intraepithelial lymphocytes are essential mediators of host-microbial homeostasis at the intestinal mucosal surface. Proc. Natl. Acad. Sci. USA 108, 8743-8748 (2011).

36. Kitamura, T. New experimental approaches in retrovirus-mediated expression screening. Int. J. Hematol. 67, 351-359 (1998).

37. Montufar-Solis, D. \& Klein, J.R. An improved method for isolating intraepithelial lymphocytes (IELs) from the murine small intestine with consistently high purity. J. Immunol. Methods 308, 251-254 (2006). 